
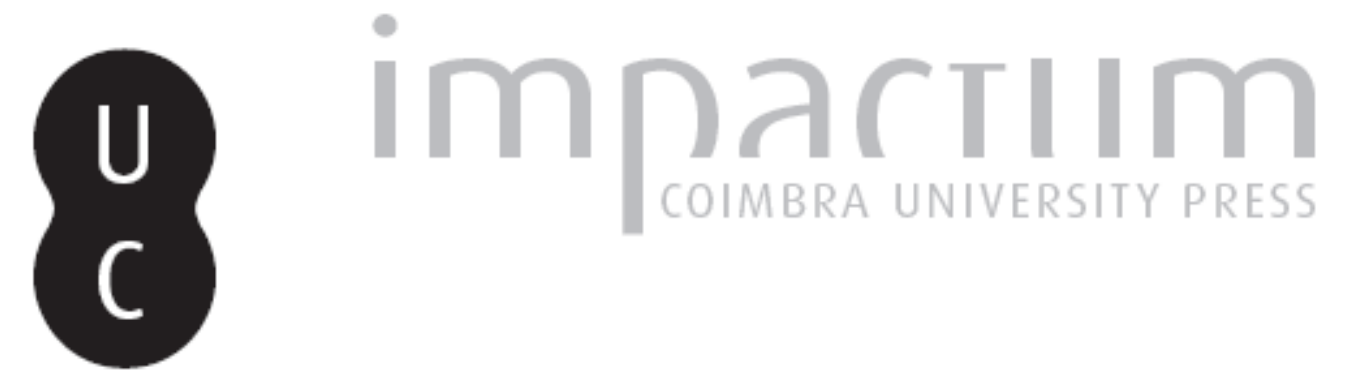

\title{
High-frequency trading (HFT)
}

\author{
Autor(es): Quelhas, José Manuel
}

Publicado por: Faculdade de Direito da Universidade de Coimbra

URL persistente:

URI:http://hdl.handle.net/10316.2/39888

DOI:

DOI:https://doi.org/10.14195/0870-4260_58_8

Accessed : $\quad$ 26-Apr-2023 13:59:18

A navegação consulta e descarregamento dos títulos inseridos nas Bibliotecas Digitais UC Digitalis, UC Pombalina e UC Impactum, pressupõem a aceitação plena e sem reservas dos Termos e Condições de Uso destas Bibliotecas Digitais, disponíveis em https://digitalis.uc.pt/pt-pt/termos.

Conforme exposto nos referidos Termos e Condições de Uso, o descarregamento de títulos de acesso restrito requer uma licença válida de autorização devendo o utilizador aceder ao(s) documento(s) a partir de um endereço de IP da instituição detentora da supramencionada licença.

Ao utilizador é apenas permitido o descarregamento para uso pessoal, pelo que o emprego do(s) título(s) descarregado(s) para outro fim, designadamente comercial, carece de autorização do respetivo autor ou editor da obra.

Na medida em que todas as obras da UC Digitalis se encontram protegidas pelo Código do Direito de Autor e Direitos Conexos e demais legislação aplicável, toda a cópia, parcial ou total, deste documento, nos casos em que é legalmente admitida, deverá conter ou fazer-se acompanhar por este aviso.

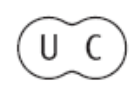




\section{UNIVERSIDADE DE COIMBRA \\ FACULDADE DE DIREITO}

\section{BOLETIMDECCÊNCIASECONÓNICAS}

VOLUME LVIII

$\begin{array}{llll}2 & 0 & 1 & 5\end{array}$

COIMBRA 


\section{HIGH-FREQUENCY TRADING (HFT)}

Nota prévia

O objectivo deste trabalho é o de avaliar o impacto sistémico da high-frequency trading (HFT) à luz do trade-off entre a eficiência e a estabilidade do mercado financeiro, ao mesmo tempo que observa a resposta regulatória da União Europeia encetada pelo Regulamento (UE) n. ${ }^{\circ}$ 596/2014 e pela Directiva 2014/65/UE.

\section{1. «Negociação algorítmica de alta frequência»}

$\mathrm{O}$ art. $^{\circ} 3 .^{\circ}$, n..$^{\circ} 1$, ponto 33 , da versão portuguesa do Regulamento (UE) n..$^{\circ}$ 596/2014, de 16 de Abril ${ }^{1}$, traduz high-

${ }^{1}$ Cfr.: Regulamento (UE) n. ${ }^{\circ}$ 596/2014 do Parlamento Europeu e do Conselho, de 16.04.2014, relativo ao abuso de mercado (regulamento abuso de mercado) e que revoga a Directiva 2003/6/CE do Parlamento Europeu e do Conselho e as Directivas 2003/124/ CE, 2003/125/CE e 2004/72/CE [versão rectificada e consolidada, CELEX 2014R0596 — PT — 02.07.2014 — 000.001]. 
-frequency trading por «negociação de alta frequência», a qual define como "técnica de negociação algorítmica de alta frequência na acepção do artigo $4 .^{\circ}$, n. $^{\circ} 1$, ponto 40, da Directiva 2014/65/UE”.

Por sua vez, o referido artigo $4 .^{\circ}$, n. $^{\circ} 1$, ponto 40 , da Directiva 2014/65/UE, de 15 de $\mathrm{Maio}^{2}$, estabelece:

"«Técnica de negociação algorítmica de alta frequência»: uma técnica de negociação algorítmica caracterizada por:

a) Uma infra-estrutura destinada a minimizar a latência de rede e de outros tipos, incluindo pelo menos um dos seguintes sistemas para a entrada de ordens algorítmicas: partilha de instalações (co-location), alojamento de proximidade ou acesso electrónico directo de alta velocidade;

b) A determinação pelo sistema da abertura, geração, encaminhamento ou execução de ordens sem intervenção humana para as transacções ou ordens individuais; e

${ }^{2}$ Cfr.: Directiva 2014/65/UE do Parlamento Europeu e do Conselho, de 15.05.2014, relativa aos mercados de instrumentos financeiros e que altera a Directiva 2002/92/CE e a Directiva 2011/61/UE, modificada pelo Regulamento (UE) n. ${ }^{\circ}$ 909/2014 do Parlamento Europeu e do Conselho, de 23.07.2014 [versão consolidada, CELEX 2014L0065 — PT — 17.09.2014 — 001.001].

A Directiva 2014/65/UE é usualmente representada pela expressão «MiFID II Directive» (Markets in Financial Instruments Directive), na sequência da «MiFID I», correspondente à Directiva 2004/39/CE do Parlamento Europeu e do Conselho, de 21.04.2004. Por sua vez, o Regulamento (UE) n. ${ }^{\circ}$ 600/2014 do Parlamento Europeu e do Conselho, de 15.05.2014, relativo aos mercados de instrumentos financeiros e que altera o Regulamento (UE) n. ${ }^{\circ}$ 648/2012, é usualmente representado pelo acrónimo «MiFIR» (Markets in Financial Instruments Regulation). De notar que, em alguns documentos da União Europeia, o acrónimo "MiFID II" corresponde ao «pacote legislativo» composto pela «MiFID II Directive» e pelo «MiFIR». Sobre o significado e a utilização dos diferentes acrónimos, cfr. FuTURES Industry Association e FIA Europe (2015). 
c) Elevadas taxas de mensagens intradiárias constituídas por ordens, ofertas de preços ou cancelamentos".

A tecnicidade desta acepção exige a interpretação individualizada de alguns dos conceitos mobilizados pela Directiva 2014/65/UE, mormente: «negociação algorítmica»; «minimização da latência»; «partilha de instalações»; «alojamento de proximidade»; «acesso electrónico directo de alta velocidade»; «ausência de intervenção humana»; «elevadas taxas de mensagens intradiárias».

Por «negociação algorítmica», o art. $^{\circ} 4^{\circ}{ }^{\circ}$, n. ${ }^{\circ} 1$, ponto 39 , da Directiva 2014/65/UE entende a

"negociação em instrumentos financeiros, em que um algoritmo informático determina automaticamente os parâmetros individuais das ordens, tais como o eventual início da ordem, o calendário, o preço ou a quantidade da ordem ou o modo de gestão após a sua introdução, com pouca ou nenhuma intervenção humana".

A referência expressa a "pouca ou nenhuma intervenção humana" é complementada pela parte final do mesmo artigo, que adita:

"Esta definição não inclui qualquer sistema utilizado apenas para fins de encaminhamento de ordens para uma ou mais plataformas de negociação, para o processamento de ordens que não envolvam a determinação de parâmetros de negociação ou para a confirmação das ordens ou o processamento pós-negociação das transacções executadas" [cfr.: art. ${ }^{\circ}$ 4. $^{\circ}$, n. $^{\circ}$ 1, ponto 39, in fine, da Directiva 2014/65/UE].

Destarte, o encaminhamento, o processamento, a confirmação e a execução de ordens por via informática - há várias décadas realizados por este meio - não bastam para a conformação de uma negociação como algorítmica; com efeito, para que uma negociação seja classificada de algorítmica exige-se que 
os próprios parâmetros de negociação - nomeadamente, o inicio, o calendário, o preço, a quantidade ou o modo de gestão das ordens - sejam determinados automaticamente por um algoritmo informático. Assim, a intervenção humana restringe-se à programação do algoritmo a montante da negociação; após a sua criação, o algoritmo «vivifica-se» e adquire uma faculdade negocial autónoma.

Em ciências da computação, «minimização da latência» ${ }^{3}$ refere o objectivo de redução do intervalo de tempo entre a transmissão e a recepção de dados informáticos, o que implica o aumento da velocidade de propagação da informação, bem como a melhoria dos respectivos meios de transferência.

Sublinhe-se que, na «técnica de negociação algorítmica de alta frequência», as unidades de medida de tempo utilizadas são o milissegundo e o microssegundo, ou seja, a prossecução do objectivo de «minimização da latência» opera à escala da milésima parte do segundo e da milionésima parte do segundo.

Neste sentido, «negociações algorítmicas de alta frequência» correspondem a «negociações algorítmicas de baixa latência», cujas operações podem ser classificadas de «baixa latência» (low latency), com duração inferior a 10 milissegundos, de «ultra baixa latência» (ultra low latency), com duração inferior a 1 milissegundo, e de «ultra ultra baixa latência» (ultra-ultra low latency), usualmente computadas em microssegundos ${ }^{4}$.

${ }^{3}$ Sobre o conceito de «latência» no domínio da «negociação algorítmica», cfr.: Hinton, BAKER e HILl (2012); HASBROUCKA e SAAR (2013); Goldstein, Kumar e Graves (2014); Bouveret, Guillaumie, Roqueiro, WinkLer e Nauhaus (2014); Gilberto (2015); O’Hara (2015).

${ }^{4}$ Sobre o alcance da diferenciação entre operações de «baixa latência», de «ultra baixa latência» e de «ultra ultra baixa latência», cfr. O’Hara (2015). 
Por «partilha de instalações» (co-location) $)^{5}$, a Directiva 2014/65/UE alude a um dos sistemas para a entrada de ordens algorítmicas, programado para a "minimização da latência», em redes de infra-estruturas informáticas de «negociação de alta frequência». A co-location consiste no alojamento de servidores informáticos dos operadores (alojados) nas instalações das bolsas de valores (alojadores) com o intuito de diminuir a distância entre os sistemas computacionais de transmissão e de recepção de ordens algorítmicas.

A diminuição da distância reduz a «latência de rede»e propicia ganhos de velocidade; embora estes ganhos sejam mensuráveis em microssegundos, a aceleração da entrada de ordens algoritmicas ocasiona vantagens competitivas consideráveis aos alojados, que «partilhem instalações» com os alojadores responsáveis pelas plataformas de negociação ${ }^{6}$.

${ }^{5}$ Sobre o impacto da co-location na «negociação algorítmica de alta frequência», cfr.: Aldridge (2013); Bouveret, Guillaumie, Roqueiro, Winkler e Nauhaus (2014); Frino, Mollica e Webb (2014); Zook e Grote (2014); Clapham, Haferkorn e ZimmerMANN (2015).

${ }^{6} \mathrm{O}$ «considerando 62» da Directiva 2014/65/UE estabelece que os alojadores prestem os "serviços de partilha das instalações de forma não discriminatória, equitativa e transparente" com o objectivo de "assegurar condições para uma negociação ordenada e equitativa". Neste sentido, o art. ${ }^{\circ} 48 .^{\circ}$, n. ${ }^{\circ} 8$, desta Directiva determina: "Os Estados-Membros exigem que os mercados regulamentados garantam que as suas regras em matéria de serviços de partilha das instalações sejam transparentes, equitativas e não discriminatórias"; por sua vez, a alínea d), do n. ${ }^{\circ} 12$, do mesmo artigo atribui à Autoridade Europeia dos Valores Mobiliários e dos Mercados competência para redigir projectos de normas técnicas de regulamentação sobre "Os requisitos para assegurar que os serviços de partilha das instalações e as estruturas de comissões sejam equitativos e não discriminatórios e que as estruturas de comissões não criem condições que contribuam para 
Por «alojamento de proximidade» (proximity hosting), a Directiva 2014/65/UE menciona outro dos sistemas para a entrada de ordens algorítmicas, programado para a «minimização da latência», em «negociação de alta frequência». Embora sejam conceitos próximos e, por vezes, utilizados indistintamente, a co-location diferencia-se da proximity hosting ${ }^{7}$. Com efeito, o «alojamento de proximidade» não significa a «partilha de instalações» entre alojados e alojadores responsáveis pelas plataformas de negociação, mas sim a locação e a utilização por parte dos operadores de servidores informáticos de terceiros (v.g., fornecedores de serviços de data center), sediados nas imediações das bolsas de valores, conquanto em espaços físicos distintos, ou em locais estratégicos de acesso a várias plataformas de negociação.

Em princípio, a co-location proporciona menor «latência» do que a proximity hosting, embora implique custos mais altos para os operadores e circunscreva as negociações a uma única plataforma; pelo contrário, a proximity hosting reduz os custos operacionais, através do outsourcing de servidores informáticos, e faculta o acesso a várias plataformas de negociação.

Por «acesso electrónico directo de alta velocidade», a Directiva 2014/65/UE refere outro dos sistemas para a entrada de ordens

perturbar a negociação ou para situações de abuso de mercado".

Em suma, embora a co-location reduza a «latência de rede»e aumente a eficiência do mercado, também pode suscitar questões de concorrência entre operadores alojados e não alojados ou alojados em condições diferentes, bem como situações de abuso de mercado, cujo combate constitui uma das incumbências das autoridades reguladoras dos mercados financeiros.

${ }^{7}$ Sobre a diferença entre co-location e proximity hosting, cfr.: CAIvano, Ciccarelli, Di Stefano, Fratini, Gasparri, Giliberti, Linciano e Tarola (2012); Merli, Massa e Lucchetti (2014). 
algoritmicas, programado para a «minimização da latência». De notar que o art. $^{\circ} 4 .^{\circ}$, n. $^{\circ} 1$, ponto 41 , da referida Directiva define «acesso electrónico directo» ${ }^{8}$ nos seguintes termos:

"um mecanismo através do qual um membro, participante ou cliente numa plataforma de negociação permite que uma pessoa utilize o seu código de negociação para que possa transmitir por via electrónica directamente à plataforma de negociação ordens relativas a um instrumento financeiro e inclui mecanismos que envolvam a utilização, por uma pessoa, da infra-estrutura do membro, participante ou cliente ou de qualquer sistema de conexão por ele disponibilizado para transmitir as ordens (acesso directo de mercado) e os mecanismos em que essa infra-estrutura não seja utilizada por uma pessoa (acesso patrocinado)".

$\mathrm{O}$ «acesso electrónio directo» compreende o «acesso directo ao mercado» [DMA (Direct Market Access)] e o «acesso patrocinado» [SA (Sponsored Access)]. Nos dois modelos, a transmissão electrónica de ordens por quem não seja «membro, participante ou cliente» de uma «plataforma de negociação» ${ }^{9}$ de instrumentos financeiros depende da autorização de utilização do «código de negociação» de quem seja «membro, participante ou cliente».

Porém, no primeiro modelo, a infra-estrutura e o sistema de conexão pertencem ao «membro, participante ou cliente»

${ }^{8}$ Sobre a noção e as modalidades de «acesso electrónico directo», cfr.: Noda (2010); IOSCO (2010); GOMBER, ARndt, LutAT e UHLE (2011); IOSCO (2015).

${ }^{9}$ Sobre a noção de «plataforma de negociação», cfr. o art. ${ }^{\circ} 4$, n. $^{\circ}$ 1, ponto 24, da Directiva 2014/65/UE, que compreende «mercados regulamentados», «sistemas de negociação multilateral» [MTF (Multilateral Trading Facilities)] e «sistemas de negociação organizados» [OTF (Organised Trading Facilities)]. Por sua vez, as definições de «mercado regulamentado», de MTF e de OTC reportam-se sucessivamente aos pontos 21,22 e 23 , do n. ${ }^{\circ} 1$, do art. ${ }^{\circ} .^{\circ}$. 
da «plataforma de negociação», ao passo que, no segundo modelo, as transmissões operam através de infra-estruturas e de sistemas de conexão dos próprios ordenantes.

Destarte, no «acesso directo ao mercado», tanto o «código de negociação» como a infra-estrutura e os sistemas de conexão são regulados pelas normas aplicáveis aos «membros, participantes ou clientes» de uma «plataforma de negociação», enquanto, no «acesso patrocinado», as infra-estruturas e os sistemas de conexão primam pela desregulação. Consequentemente, o sponsored access permite que os ordenantes individualizem as infra-estruturas e os meios de conexão, no intuito de obterem vantagens comparativas, mormente a da aceleração da entrada de ordens algorítmicas, nas operações de «ultra baixa latência» e de «ultra ultra baixa latência».

A conjugação da desregulação de infra-estruturas e de sistemas de conexão com a aceleração das operações aumenta a volatilidade dos mercados e suscita problemas de instabilidade sistémica; com efeito, os investidores/ordenantes - que não são «membros, participantes ou clientes» de uma «plataforma de negociação» - acedem a essa «plataforma de negociação», através de um «código de negociação» cedido por um «membro, participante ou cliente» dessa «plataforma de negociação», ao mesmo tempo que utilizam, particularizam e renovam as suas próprias infra-estruturas e sistemas de conexão, o que aumenta a entropia nos mercados financeiros.

Por «ausência de intervenção humana», a Directiva 2014/65/UE compreende a determinação automática dos parâmetros de negociação dos instrumentos financeiros por algoritmos informáticos ${ }^{10}$, o que configura o império da automated

${ }^{10}$ Sobre a evolução e o impacto da utilização de algoritmos informáticos nos mercados financeiros, cfr.: THe GOVERNMENT OF- 
finance sobre a behavioural finance ${ }^{11}$, traduzido na prevalência do computer trading sobre o buman trading ${ }^{12}$ ou dos machine-intermediated markets sobre os human-intermediated markets ${ }^{13}$.

A preponderância dos machine-intermediated markets em relação aos human-intermediated markets é confirmada pelo volume de transacções, nas praças financeiras mais significativas. De acordo com os dados de The Government OfFice for SCIENCE (2012, p. 19), o computer-based trading (CBT) ${ }^{14}$ representa cerca de 30\% das operações bolsistas no Reino Unido e mais de 60\% nos Estados Unidos ${ }^{15}$.

fice for Science (2012); Kumiega e Van Vliet (2012); Kirilenko (2013); Scholtus, van Dijk e Frijns (2014); Kunz e Martin (2015).

${ }^{11}$ Sobre o confronto entre automated finance e behavioural finance, cfr: Kumiega e Van Vliet (2012); Van Vliet (2012); Davis, Kumiega e VAn Vliet (2013); Johansson (2013).

${ }_{12}$ Sobre o confronto entre computer trading e buman trading, cfr.: Cliff, Brown e Treleaven (2011); Cliff e Northrop (2011); De Luca, Szostek, Cartlidge e Cliff (2011); Farmer e Skouras (2011); Hendershott (2011); Mitra, diBartolomeo, Banerjee e Yu (2011); Zigrand, Shin e Beunza (2011); Cartlidge e ClifF (2012); Johnson e Zhao (2012); The Government Office for SCIENCE (2012).

${ }^{13}$ Sobre o confronto entre os buman-intermediated markets e os machine-intermediated markets, cfr.: ANGeL (2011); Menkveld (2011); The Government Office for Science (2012).

${ }^{14}$ Sobre a noção de computer-based trading (CBT), cfr. THE Government Office for Science (2012, p. 20), bem como a taxonomia apresentada em Friederich e PAYNe (2011, pp. 6-8).

${ }^{15}$ The Government Office for SCIEnCE (2012, p. 19) alerta para as dificuldades de cômputo rigoroso do volume de transacções em CBT por força da indeterminação do conceito de computer-based trading, do aceleramento da inovação tecnológica e da diversidade de estratégias negociais seguidas pelos operadores. Não obstante, THE GOVERnMENT OFFICE FOR SCIENCE (2012) ratifica as estimativas de Izabella Kaminska, divulgadas online nas páginas do FT Alphaville's Markets Live, do Financial Times, em 2009 e 2011 - cfr.: Kaminska (2009) e Kaminska (2011). 
Se é certo que a proporção do CBT no total das operações aumenta a liquidez do mercado e comprime os spreads entre os bid and ask prices ${ }^{16}$, também é verdade que a velocidade das negociações dissocia as transacções financeiras das transacções reais e suscita questões de estabilidade sistémica, tanto mais que os machine-intermediated markets assentam na desintermediação humana.

Ao contrário dos «intermediários financeiros especializados» (market makers) ${ }^{17}$, cuja actividade é regulada e supervisionada, os machine-intermediated markets supõem a desintermediação humana e a realização de transacções à «velocidade da luz», só inteligíveis "When Finance Meets Physics"18.

${ }^{16}$ Sobre as noções de bid price, ask price e spread bid-ask, cfr. QuelHAs (2012c, nota 83).

${ }_{17}$ Sobre a noção e o papel dos market makers, cfr. QuELHAs (2012c), especialmente em "1.1.7.2. A acção dos market makers".

${ }^{18}$ Sob o título elucidativo de "When Finance Meets Physics: The Impact of the Speed of Light on Financial Markets and their Regulation", ANGEL (2014) reflecte sobre as consequências da aceleração das transacções financeiras na regulação dos mercados, através da analogia com os efeitos da teoria da relatividade restrita de Einstein sobre a física newtoniana.

Neste sentido, ANGel (2014) sustenta que algumas estratégias financeiras tradicionais - v.g., arbitragem, «criação de mercado» (market-making) e «reacção a notícias» (reacting to news) - assumem novas formas e novos resultados, quando praticadas à «velocidade da luz», o que implica a transição de uma «escala de tempo humana» para uma «escala de tempo computacional», com as consequentes alterações nos métodos de regulação e de supervisão.

De um modo especial, Angel (2014) examina alguns dos objectivos da regulação dos mercados financeiros - defesa do consumidor, equidade, afectação de recursos, eficiência económica, formação de capital, solidez das instituições, estabilidade económica - à luz das transacções algorítmicas de alta frequência, sublinhando que esses objectivos podem tornar-se conflituantes, por força das 
Por «elevadas taxas de mensagens intradiárias», a Directiva 2014/65/UE não apresenta uma quantificação numérica concernente à ratio entre as ordens, ofertas de preços ou anulações e o volume de transacções intradiário.

Contudo, o «considerando 61» da Directiva 2014/65/ UE esclarece:

"Especialmente, uma negociação algorítmica de alta frequência pode conter elementos tais como a abertura, geração, encaminhamento e execução de ordens que são determinados pelo sistema sem intervenção humana para cada transacção ou ordem individual, um período curto para o estabelecimento e a liquidação de posições, uma elevada taxa de rotação da carteira, um elevado rácio ordens/transacções intradiário e o encerramento de um dia de transacções com uma posição nula ou próxima desse valor. A negociação algorítmica de alta frequência caracteriza-se, nomeadamente, por elevadas taxas de mensagens intradiárias constituídas por ordens, ofertas de preços ou anulações. Ao determinar o que se entende por elevadas taxas de mensagens intradiárias, haverá que ter em conta a identidade do cliente efectivo subjacente à actividade, a duração do período de observação, a comparação com a actividade global do mercado durante esse período e a concentração ou fragmentação relativas da actividade".

De sublinhar que a subida do rácio ordens/transacções intradiário significa uma elevada taxa de rotação da carteira de um cliente, a qual retrata um periodo curto para o estabelecimento e a liquidação de posições, bem como a tendência para o encerramento de um dia de transacções com uma posição nula ou próxima desse valor, o que implica elevadas taxas de mensagens intradiárias.

Apesar de o texto da Directiva 2014/65/UE do Parlamento Europeu e do Conselho, de 15.05.2014, não quantificar o entendimento de «elevadas taxas de mensagens intradiárias»,

diferenças entre as velocidades de negociação dos vários operadores, mesmo quando aferíveis em microssegundos. 
o ESMA's Technical Advice to the Commission on MiFID II and Mi$F R^{19}$, de 19.12.2014, recomenda a adopção de um de três critérios $^{20}$, na aprovação de «actos delegados» pela Comissão ${ }^{21}$, a saber: i) limiar absoluto por instrumento financeiro; ii) limiar absoluto por plataforma de negociação e por instrumento financeiro; iii) limiar relativo.

O primeiro critério considera a realização de «elevadas taxas de mensagens intradiárias» quando um participante, membro ou cliente de uma plataforma de negociação emita, em média, duas ordens, ofertas de preços ou anulações por segundo, em cada dia de transacção, relativas ao mesmo instrumento financeiro. Assim, se uma bolsa de valores funcionar ininterruptamente durante oito horas e meia, o mesmo cliente emitirá mais de 61200 ordens, ofertas de preços ou anulações sobre o mesmo instrumento financeiro, por dia de negociação.

O segundo critério considera a realização de «elevadas taxas de mensagens intradiárias» quando um participante, membro ou cliente de uma plataforma de negociação emita, em média, quatro ordens, ofertas de preços on anulações por segundo, em cada dia de transacção, relativas a qualquer instrumento financeiro, ou duas ordens, ofertas de preços ou anulações por segundo, em cada dia de transacção, relativas ao mesmo instrumento financeiro. Assim, se uma bolsa de valores funcionar ininterruptamente durante oito horas e meia, o mesmo cliente emitirá, por dia de negociação, mais de 122400 ordens, ofertas de preços

${ }^{19}$ Cfr. European Securities and Markets Authority (2014).

${ }^{20}$ Cfr.: European Securities and Markets Authority (2014, pp. 338-339); Futures Industry Association e FIA Europe (2015, p. 4).

${ }^{21}$ Sobre a origem, a noção e o alcance da nova tipologia de actos não legislativos denominados «actos delegados», introduzidos pelo art. ${ }^{\circ} 290 .^{\circ}$ do Tratado de Lisboa, cfr. Tenreiro e FrançA (2012, pp. 1039-1043). 
ou anulações sobre qualquer instrumento financeiro ou mais de 61200 ordens, ofertas de preços ou anulaçoes sobre o mesmo instrumento financeiro.

O terceiro critério considera a realização de «elevadas taxas de mensagens intradiárias» quando a duração média diária das ordens, ofertas de preços ou anulações, emitidas por um participante, membro ou cliente de uma plataforma de negociação, seja inferior à duração média diária de todas as ordens, ofertas de preços ou anulaçoes, emitidas por todos os participantes, membros ou clientes da mesma plataforma de negociação.

A European Securities and Markets Authority propõe a classificação de «elevadas taxas de mensagens intradiárias» quando a duração média diária das ordens, ofertas de preços ou anulações, emitidas por um participante, membro ou cliente de uma plataforma de negociação, fique aquém de um limiar a definir compreendido entre os percentis 20 e 40 da duração média diária de todas as ordens, ofertas de preços ou anulações, emitidas por todos os participantes, membros ou clientes da mesma plataforma de negociação.

Independentemente do critério que os «actos delegados» da Comissão venham a consagrar, as três propostas do ESMA's Technical Advice to the Commission on MiFID II and MiFIR enfatizam a rotação dos activos transaccionados e a primazia pelo ultra curto prazo. Destarte, a rotação das carteiras e o short-termism ${ }^{22}$ das operações são inerentes às negociações algorítmicas de alta frequência ou, com mais rigor, a sobrerrotação das carteiras e o ultra short-termism constituem elementos essenciais destas transacções, o que reforça a questão da instabilidade sistémica.

Acresce que a emissão diária de múltiplas ordens de

22 Sobre o significado e o impacto do short-termism nos mercados financeiros, cfr.: Dallas (2012); Davies, Haidane, NiEIsen e Pezzini (2014). 
abertura e de fecho de posições sobre os mesmos activos subjacentes pode desencadear o encerramento de um dia de transaccooes com uma posição nula ou próxima desse valor ${ }^{23}$, o que sublinha a natureza especulativa das negociações algorítmicas de alta frequência. Com efeito, a composição relativa das carteiras, no início e no final do dia, pode ser idêntica, apesar das múltiplas transacções de compra e de venda entretanto realizadas nesse mesmo dia.

\section{2. «Too fast to fail»}

As características da «negociação algorítmica de alta frequência», observadas no ponto 1, estimulam o debate acerca do trade-off entre a eficiência e a estabilidade do sistema financeiro ${ }^{24}$. Porém, o crescimento da high-frequency trading desenhou um novo cenário nos mercados financeiros, retratado por LEWIS (2014, p. 13), nos seguintes termos:

${ }^{23}$ Sobre diferentes perspectivas acerca do impacto da adopção da estratégia de flat position (posição nula) no fecho das negociações diárias, cfr.: Gomber, Arndt, LutAT e UhLE (2011); SORnEtTE e von der Becke (2011); Benos e Sagade (2012); Keller (2012).

${ }^{24}$ Sobre o trade-off entre a eficiência e a estabilidade do sistema financeiro, cfr.: QuELHAS (2012a), especialmente em "2. A evitação do «efeito de contágio»"; Quelhas (2012c), sobretudo em "1.1.7.3. A acção dos investidores institucionais", "1.1.8.3. Os modelos de ataques especulativos de "terceira geração»" e "1.2. Sobre a diversidade de contributos para a compreensão das crises financeiras"; QuelHas (2013a), particularmente em "2.4. A interconexão entre os bancos europeus". 
"Na última década os mercados financeiros mudaram tão depressa que a imagem que temos deles deixou de ser real. Imaginamos as cotações de bolsa a desfilar na parte de baixo dos ecrãs de televisão e vemos machos-alfa vestidos com coletes coloridos, cada cor a corresponder a um código, a gritarem uns para os outros na sala de negociação. Esta fotografia está datada e o mundo que representa está morto. Desde 2007 que não existem tipos ferozes e de coletes coloridos na bolsa. $\mathrm{Ou}$ se existem não têm razão de ser. Ainda há alguns seres humanos a trabalhar na Bolsa de Nova Iorque e nas várias bolsas de Chicago, mas já não decidem nada em nenhum mercado financeiro, nem têm acesso privilegiado aos mesmos. O mercado de acções americano agora negoceia-se dentro de caixas negras, em edifícios fortemente protegidos em Nova Jérsia ou Chicago. O que acontece dentro dessas caixas negras é difícil de saber - as cotações que desfilam nos ecrãs de televisão representam apenas uma fracção ínfima do que se passa nos mercados de valores".

Ora, este novo cenário reconfigura o aludido trade-off entre a eficiência e a estabilidade do sistema financeiro, suscitando novas questões ou reformatando as antigas. De notar que a profusão da «negociação algorítmica de alta frequência» já ocorre após a deflagração da crise financeira de $2007^{25}$, ou

${ }^{25}$ Sobre a origem, o significado e os efeitos da crise financeira de 2007, cfr.: QueLHAS (2012a) sobretudo em "1. O reforço da resiliência do sistema financeiro", "4. A contenção do financiamento público das operações de bailout" e "5. O progresso da coordenação supervisora para a harmonização decisória”; QuelHAs (2012b) particularmente em "1. O "pacote legislativo» de 24 de Novembro de 2010", "2. Monitorização e avaliação do risco sistémico" e "4. Supervisão sistémica e efeito de contágio"; QuelHAs (2013a) especialmente em "1.1. Redução do risco sistémico", "1.2. Redução do risco de contágio", "1.3. Redução do risco moral", "1.5. Manutenção da integridade do mercado interno", "2.1. A utilização de instrumentos financeiros complexos", "2.2. A preponderância do financiamento de curto prazo" e "2.3. A inadequação dos me- 
seja, aos problemas subsequentes à crise do subprime ${ }^{26}$ acrescem os inerentes ao bigh-frequency trading.

A imperiosidade da reconfiguração do trade-off entre a eficiência e a estabilidade do sistema financeiro deriva principalmente da aceleração das transacções e da utilização de «caixas negras» ${ }^{27}$. Com efeito, antes da difusão da «negociação algorítmica de alta frequência», o trade-off pressupunha o compromisso entre o progresso da inovação financeira, propiciador de eficiência, desenvolvido pelos operadores de mercado, e a moderação da inovação financeira, propiciadora de estabilidade, assegurada pelos reguladores e supervisores Após a proliferação da «negociação algorítmica de alta frequência», a aceleração das transacções e a sua automação corroem os pressupostos do referido trade-off porque a realização de operações à «velocidade da luz» significa a transição de uma «escala de tempo humana» para uma «escala de tempo computacio-

canismos de protecção dos depositantes"; QuelHAs (2013b) mormente em "5.1. A cura de austeridade e o "risco moral»" e "5.2. A cura de austeridade e a internalização de custos"; QuelHAs (2014), principalmente em "2. O carácter precursor do Decreto-Lei n. ${ }^{\circ} 31-\mathrm{A} / 2012$, de 10 de Fevereiro".

${ }^{26}$ Sobre o sentido da expressão subprime, cfr. AleXANDRE, MARTINS, ANdrade, Castro e BaÇão (2009) e Quelhas (2013a, pp. 503-507).

${ }^{27}$ A expressão «caixas negras» simboliza «supercomputadores», dotados de elevadíssima capacidade de processamento de informação e de armazenamento de dados, aptos a executarem algoritmos complexos de alta frequência, com velocidades mensuráveis em milissegundos $\left(10^{-3}\right)$, microssegundos $\left(10^{-6}\right)$ e nanossegundos $\left(10^{-9}\right)$. LEWIS (2014, p. 68) descreve o mercado de valores americano como "um sistema de castas baseado na velocidade", contrapondo a casta dos que que podem comprar nanossegundos com a casta dos que não podem comprar nanossegundos; a primeira casta compreende a vantagem da velocidade e tem uma visão perfeita do mercado; a segunda casta nem sequer se apercebe do valor do nanossegundo e não descortina o mercado. 
nal» $\rangle^{28}$, o que impede ou dificulta a obtenção de compromissos entre a inovação e a segurança dos mercados, bem como a monitorização das «caixas negras» dos operadores por reguladores e supervisores financeiros.

No artigo "Too fast to fail: are high-speed traders hurtling toward the next financial meltdown?”, BAUMANN (2013) alerta para a possibilidade de a negociação de milhares de títulos em escassos nanossegundos causar a deflagração de «crises financeiras sistémicas» ${ }^{29}$.

Com efeito, a aceleração e o incremento das operações algorítmicas de alta frequência evidenciam o desfasamento entre a realidade dos mercados financeiros e os respectivos mecanismos de regulação e de supervisão. Destarte, aos aforismos «too big to fail $\rangle^{30}$, «too important to fail $\rangle^{31}$, «too many to fail $\rangle^{32}$, «too similar to fail $\rangle^{33}$, «too connected to fail $\rangle^{34}$, junta-se a máxima «too fast to fail».

${ }^{28}$ Cfr., supra, nota 18.

${ }^{29}$ Sobre o debate em torno da noção e do impacto de «crises financeiras sistémicas», cfr. QuelHAs (2012c), principalmente o "Capítulo 3. Do risco sistémico e do «efeito de contágio»: São as crises financeiras sistémicas?”.

30 Sobre o significado e o enquadramento do aforismo «too big to fail», cfr.: QuelHAs (2012a, pp. 247-248); QuELHAS (2013a, pp. 526-535); DORN (2015a, p. 28 e ss); DORN (2015b, pp. 209-234).

${ }^{31}$ Sobre a acepção do aforismo «too important to fail», cfr. DORN (2015a, pp. 78-81).

32 Sobre a origem e o alcance do aforismo "too many to fail», cfr.: Acharya e Yorulmazer (2007); Litkanen, Bänziger, Campa, Gallois, Goyens, Krahnen, Mazzucchelli, Sergeant, Tuma, VaNHEVEL e WijfFELS (2012, pp. 32-38); QuelHas (2013a, pp. 526-535).

33 Sobre a interpretação do aforismo «too similar to fail», cfr.: Quelhas (2013a, pp. 526-535); Dorn (2015a, pp. 39-42); DORN (2015b, pp. 209-234).

34 Sobre o sentido e as consequências do aforismo «too connected to faill, cfr.: Dorn (2015a, p. 28 e ss); DORN (2015b, pp. 209-234). 
LEWIS (2014, pp. 99-100) descreve a mutação impetuosa da realidade dos mercados financeiros, nos seguintes termos:

"O mercado totalmente informatizado evoluía demasiado depressa para que os humanos pudessem intervir e, por isso, ali estavam os traders de alta frequência. A sua importância podia ser inferida pela sua actividade. Em 2005, as firmas de HFT faziam 25\% de todas as transacções nos mercados públicos de valores. Em 2008, esse número subiu para $65 \%$. Esta nova tendência do mercado - argumentavam os defensores - era um sinal de progresso, não apenas necessário, mas também positivo para os investidores. Antes, quando os humanos intermediavam o mercado, o spread entre as licitações e as ofertas de uma acção eram 1/16 de um ponto percentual. Agora que os computadores faziam o trabalho, pelo menos nas acções mais transaccionadas, essa diferença era de $1 / 100$ de um ponto percentual. Os defensores das transacções de alta frequência alegavam, por isso, que mais HFT significava mais liquidez".

De sublinhar que LEWIS (2014) compara dados anteriores à deflagração da crise do subprime com dados posteriores, ou seja, apesar da contracção drástica da economia real, a proporção das transacções algorítmicas de alta frequência aumentou de $25 \%$ para $65 \%$ do total das transacções bolsistas, ao mesmo tempo que os spreads dos traders de alta frequência baixaram de $0,0625 \%$ para $0,01 \%$, o que propiciaria o aumento geral da liquidez.

Contudo, o incremento do volume de transacções bolsistas e a contracção dos spreads dos traders não garantem, per se, o aumento da liquidez. Como denota LEwIs (2014, p. 100) não se deve confundir «actividade de transacções» com «liquidez», tanto mais que o short-termism, o ultra-short-termism, a sobrerrotação das carteiras e a adopção diária de «posições nulas», no fecho dos mercados, imperam na «negociação algorítmica de alta frequência» - cfr. supra ponto 1 .

De facto, transacções sucessivas e ultra-rápidas sobre 
os mesmos activos subjacentes às operações algorítmicas de alta frequência empolam a actividade dos mercados financeiros, mas não aumentam a liquidez disponível para a economia real. Acresce que a descida abrupta dos spreads dos traders de alta frequência foi compensada pela subida expressiva do volume de transacções, ou seja, os proveitos dos traders não diminuíram, apesar da contracção significativa da economia real, após a crise do subprime.

No mesmo sentido, LEWIS (2014, p. 104) acrescenta:

"Desde 2005, a dimensão média de cada transacção no mercado americano caíra a pique, os mercados estavam fragmentados e alargara-se o fosso entre a visão do público sobre os mercados e a visão que deles tinham os traders de alta frequência. $\mathrm{O}$ crescimento das transacções de alta frequência foi acompanhado, em paralelo, pelo aumento da volatilidade do mercado de valores, mesmo sem contar com o turbilhão causado pela crise financeira de 2008. A volatilidade do preço de cada transacção no mercado americano, entre 2010 e 2013, foi cerca de $40 \%$ mais alta do que no período entre 2004 e 2006. Houve dias em $2011 \mathrm{em}$ que a volatilidade foi maior do que quando rebentou a bolha das empresas «ponto com» [no ano de 2000]".

Nestes termos, o aumento do volume de transacções e a contracção dos spreads dos traders de alta frequência foram acompanhados pela diminuição da dimensão média de cada transacção e pela fragmentação dos mercados, o que alarga o fosso entre a casta dos que podem comprar nanossegundos e a casta dos que não podem comprar nanossegundos ${ }^{35}$.

A diminuição da dimensão média de cada transacção justifica-se pela estratégia de fraccionamento das operações bolsistas de grande dimensão em múltiplas transacções algorítmicas de pequena dimensão, com baixos spreads unitários, mas de elevadíssima velocidade de negociação e intensíssima rotativida-

${ }^{35}$ Cfr., supra, nota 27. 
de de activos, capazes de arbitrarem diferenças infinitesimais entre variações ultra-rápidas de cotações, no mesmo mercado ou em mercados diferentes.

Por sua vez, a fragmentação dos mercados justifica-se pelo desenvolvimento e aproveitamento de oportunidades de arbitragem, decorrentes da «minimização da latência» das transacções entre diferentes mercados bolsistas, bem como pela proliferação de plataformas alternativas, usualmente privadas e anónimas, de negociação algorítmica fora de bolsa, usualmente designadas por dark pools ${ }^{36}$.

A expressão dark pools generalizou-se na terminologia financeira no sentido de plataformas electrónicas alternativas de negociação algorítmica de valores mobiliários, fora das bolsas oficiais, normalmente de natureza privada e utilizadas por investidores institucionais, caracterizadas pela opacidade das transacções, pelo anonimato dos contratantes e pela velocidade das operações.

A opacidade das transacções traduz-se pela não divulgação das cotações e dos volumes dos activos negociados. O anonimado dos contratantes manifesta-se pela não publicitação dos investidores e dos concernentes compromissos assumidos no mercado. A velocidade das operações exprime-se através da sua concretização em milissegundos, microssegundos e nanossegundos.

Estas características acentuam a volatilidade dos mercados financeiros, denunciada por LEwis (2014, p. 104), quando sustenta que a volatilidade média das cotações dos activos

${ }^{36}$ Sobre a evolução, as características, a diversidade, a velocidade de negociação e o papel das dark pools nos mercados financeiros, bem como as dificuldades e os desafios que suscitam às autoridades de regulação e de supervisão, cfr.: PATterson (2013); BANKs (2014); Niciejewska (2015). 
transaccionados aumentou a par do crescimento da «negociação algorítmica de alta frequência», o que recoloca a questão do seu impacto sobre a estabilidade sistémica ${ }^{37}$.

\section{Nota final}

Em conclusão, o trade-offentre a eficiência e a estabilidade do sistema subjaz às preocupações teleológicas da legislação europeia recente sobre mercados e instrumentos financeiros, mormente da Directiva 2014/65/UE.

De um modo especial, a «negociação algorítmica de alta frequência» questiona as estruturas de regulação e de supervisão dos mercados financeiros globais, particularmente as da União Europeia, que continuarão a ser objecto de reflexão e de debate no futuro próximo.

${ }^{37}$ Sobre o impacto sistémico da «negociação algorítmica de alta frequência», cfr.: Bloomfield e WetheriLt (2012); DanieLsSON e ZER (2012); Hu (2012). 


\section{Referências Bibliográficas}

Acharya, Viral V. e Yorulmazer, Tanju (2007): “Too many to fail - An analysis of time-inconsistency in bank closure policies", em Journal of Financial Intermediation, Vol. 16, n. ${ }^{\circ}$ 1, Janeiro, pp. 1-31.

Aldridge, Irene (2013): High-Frequency Trading: A Practical Guide to Algorithmic Strategies and Trading Systems, 2. ${ }^{\mathrm{a}}$ ed., Hoboken, John Wiley and Sons.

Alexandre, Fernando, Martins, Ives Gandra, Andrade, João Sousa, Castro, Paulo Rabello e BAÇão, Pedro (2009): A Crise Financeira Internacional, Coimbra, Imprensa da Universidade de Coimbra.

AngEL, James J. (2011): "Impact of special relativity on securities regulation", Foresight Driver Review, n. ${ }^{\circ}$ DR 15, 20 de Abril, Londres, UK Government's Foresight Project, The Future of Computer Trading in Financial Markets, Government Office for Science, 15 pp. [formato electrónico disponivel em <www.foresight.gov.uk>].

- (2014): "When finance meets physics: the impact of the speed of light on financial markets and their regulation", em The Financial Review, Vol. 49, n. ${ }^{\circ}$ 2, Maio pp. 271-281.

Banks, Erik (2014): Dark Pools: Off-Exchange Liquidity in an Era of High Frequency, Program, and Algorithmic Trading, 2. ${ }^{\mathrm{a}}$ ed., Basingstoke, Palgrave Macmillan.

Baumann, Nick (2013): “Too fast to fail: are high-speed traders hurtling toward the next financial meltdown?", em $\mathrm{Mo}$ ther Jones, Vol. 38, n. ${ }^{\circ}$ 1, Janeiro/Fevereiro, p. 36 [formato electrónico disponível em <http://www.motherjones. $\mathrm{com} /$ politics / 2013/02/high-frequency-trading-danger-risk-wall-street>, sob o título "Too Fast to Fail: Is High-Speed Trading the Next Wall Street Disaster?"].

Benos, Evangelos e SAGade, Satchit (2012): "High-frequency trading behaviour and its impact on market quality: evidence from the UK equity market", Bank of England Working Papers, n. ${ }^{\circ}$ 469, Dezembro, Londres, Bank of England, 30 pp. 
Bloomfield, Robin e Wetherilt, Anne (2012): "Computer trading and systemic risk: a nuclear perspective", Foresight Driver Review, n. ${ }^{\circ}$ DR 26, 23 de Outubro, Londres, UK Government's Foresight Project, The Future of Computer Trading in Financial Markets, Government Office for Science, 76 pp. [formato electrónico disponível em $<$ www.foresight.gov.uk>].

Bouveret, Antoine, Guillaumie, Cyrille, Roqueiro, Carlos Aparicio, Winkler, Christian e Nauhaus, Steffen (2014): "High-frequency trading activity in EU equity markets", ESMA Economic Report, n. ${ }^{\circ}$ 1, Paris, European Securities and Markets Authority, 31 pp.

Caivano, Valeria, Ciccarelli, Salvatore, Di Stefano, Giovanna, Fratini, Marco, Gasparri, Giorgio, Giliberti, Monica, Linciano, Nadia e TAROLA, Isadora (2012): "Il trading ad alta frequenza - Caratteristiche, effetti, questioni di policy", CONSOB Discussion Papers, n. ${ }^{\circ}$ 5, Dezembro, Roma, Commissione Nazionale per le Società e la Borsa, $57 \mathrm{pp}$. [formato electrónico disponível em <SSRN: http:/ /ssrn. com/abstract $=2191669>$ ].

Cartlidge, John e ClifF, Dave (2012): "Exploring the "robot phase transition" in experimental human-algorithmic markets", Foresight Driver Review, n. ${ }^{\circ}$ DR 25, 2 de Abril, Londres, UK Government's Foresight Project, The Future of Computer Trading in Financial Markets, Government Office for Science, 50 pp. [formato electrónico disponível em $<$ www.foresight.gov.uk>].

Clapham, Benjamin, Haferkorn, Martin e Zimmermann, Kai (2015): "Does Speed Matter? The Role of High-Frequency Trading for Order Book Resiliency", Chair of e-Finance, Maio, Frankfurt, Goethe University, Faculty of Economics and Business Administration, 28 pp. [formato electrónico disponível em SSRN: <http://ssrn.com/ abstract $=2603205>$ ]. 
Cliff, Dave, Brown, Dan e Treleaven, Philip (2011): “Technology trends in the financial markets: A 2020 vision”, Foresight Driver Review, n. ${ }^{\circ}$ DR 3, 8 de Setembro, Londres, UK Government's Foresight Project, The Future of Computer Trading in Financial Markets, Government Office for Science, 48 pp. [formato electrónico disponível em www. foresight.gov.uk $>$ ].

ClifF, Dave e Northrop, Linda (2011): “The Global Financial Markets: an Ultra-Large-Scale Systems perspective", Foresight Driver Review, n. ${ }^{\circ}$ DR 4, 8 de Outubro, Londres, UK Government's Foresight Project, The Future of Computer Trading in Financial Markets, Government Office for Science, 48 pp. [formato electrónico disponível em $<$ www.foresight.gov.uk $>$ ].

DalLas, Lynne (2012): "Short-Termism, the Financial Crisis, and Corporate Governance”, Legal Studies Research Paper Series, n. ${ }^{\circ} 12-078$, Fevereiro, San Diego, University of San Diego, School of Law, 101 pp. [formato electrónico disponível em <SSRN: http://ssrn.com/abstract=2006556>].

Danielsson, Jon e Zer, Ilknur (2012): "Systemic risk arising from computer based trading and connections to the empirical literature on systemic risk", Foresight Driver Review, n. ${ }^{\circ}$ DR 29, 23 de Outubro, Londres, UK Government's Foresight Project, The Future of Computer Trading in Financial Markets, Government Office for Science, 18 pp. [formato electrónico disponível em <www.foresight.gov.uk>].

Davies, Richard, Haldane, Andrew G., Nieisen, Mette e Pezzini, Silvia (2014): "Measuring the costs of short-termism", em Journal of Financial Stability, Vol. 12, n. ${ }^{\circ}$ 1, Junho, pp. 16-25.

Davis, Michael, Kumiega, Andrew e Van Vliet, Benjamin Edward (2013): "Ethics, Finance, and Automation: A Preliminary Survey of Problems in High Frequency Trading", em Science and Engineering Ethics, Vol. 19, n. ${ }^{\circ}$ 3, pp. 851-874. 
De Luca, Marco, Szostek, Charlotte, Cartlidge, John e Cliff, Dave (2011): "Studies of interactions between human traders and Algorithmic Trading Systems", Foresight Driver Review, n. ${ }^{\circ}$ DR 13, 8 de Setembro, Londres, UK Government's Foresight Project, The Future of Computer Trading in Financial Markets, Government Office for Science, 60 pp. [formato electrónico disponível em <www. foresight.gov.uk $>$ ].

Dorn, Nicholas (2015a): Democracy and Diversity in Financial Market Regulation, Abingdon, Routledge.

- (2015b): "Financial Markets and Regulatory Accountability: Between Technocratic Autonomy and Democratic Direction", em Accountability and Regulatory Governance - Audiences, Controls and Responsibilities in the Politics of Regulation, org. por Bianculli, Andrea C., Fernández-I-Marín, Xavier e Jordana, Jacint, Basingstoke, Palgrave Macmillan.

European Securities and Markets Authority (2014): "Final Report - ESMA's Technical Advice to the Commission on MiFID II and MiFIR", n. ${ }^{\circ}$ ESMA/2014/1569, 19 de Dezembro, Paris, European Securities and Markets Authority, 446 pp. [formato electrónico disponível em <www. esma.europa.eu>].

Farmer, J. Doyne e Skouras, Spyros (2011): "An ecological perspective on the future of computer trading", Foresight Driver Review, n. ${ }^{\circ}$ DR 6, 21 de Agosto, Londres, UK Government's Foresight Project, The Future of Computer Trading in Financial Markets, Government Office for Science, 40 pp. [formato electrónico disponível em $<$ www.foresight.gov.uk $>$ ].

Friederich, Sylvain e Payne, Richard (2011): "Computer based trading, liquidity and trading costs", Foresight Driver Review, n. ${ }^{\circ}$ DR 5, 31 de Agosto, Londres, UK Government's Foresight Project, The Future of Computer Trading in Financial Markets, Government Office for Science, 39 pp. [formato electrónico disponível em <www.foresight.gov.uk>].

Frino, Alessandro, Moldica, Vito e WebB, Robert I. (2014): “The Impact of Co-Location of Securities Exchanges' and Traders' Computer Servers on Market Liquidity", em Journal Of Futures Markets, Vol. 34, n.o 1, Janeiro, pp. 20-33. 
Futures Industry Association e FIA Europe (2015): “Algorithmic and High Frequency Trading", FIA and FIA Europe Special Report Series - Part Four, 18 de Fevereiro, Londres, FIA e FIA Europe, 9 pp. [formato electrónico disponível em $<$ https:/ / europe.fia.org/articles/special-report-series-part-four-algorithmic-and-high-frequency-trading $>$ ].

Gilberto, Fernando (2015): Negociação Algorítmica de Alta Frequência - Negócios à velocidade da luz, Porto, Vida Económica.

Goldstein, Michael A., Kumar, Pavitra e Graves, Frank C. (2014): "Computerized and High-Frequency Trading", em The Financial Review, Vol. 49, n. ${ }^{\circ}$ 2, Maio, pp. 177-202.

Gomber, Peter, Arndt, Björn, Lutat, Marco e Uhle, Tim (2011): "High-Frequency Trading", Chair of e-Finance, Junho, Frankfurt, Goethe University, Faculty of Economics and Business Administration, 86 pp. [formato electrónico disponível em <SSRN: http://ssrn.com/abstract $=1858626>$ ].

Hasbroucka, Joel e SAAr, Gideon (2013): "Low-latency trading”, em Journal of Financial Markets, Vol. 16, n. ${ }^{\circ}$ 4, pp. 646-679.

Hendershot'T, Terrence (2011): "High frequency trading and price efficiency", Foresight Driver Review, n. . DR 12, 3 de Agosto, Londres, UK Government's Foresight Project, The Future of Computer Trading in Financial Markets, Government Office for Science, 20 pp. [formato electrónico disponível em <www.foresight.gov.uk>].

Hinton, Paul, Baker, Edwin e Hill, Chris (2012): "Latency - Time for lawyers to get up to speed?", em Computer Law \& Security Review: the international journal of technology law and

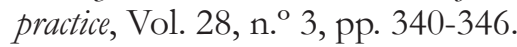

Hu, Henry T.C. (2012): "Efficient Markets and the Law: A Predictable Past and an Uncertain Future", em Annual Review Of Financial Economics, Vol. 4, pp. 179-214.

IOSCO (2010): "Principles for Direct Electronic Access to Markets", Final Report of Technical Committee of the International Organization of Securities Commissions, n. ${ }^{\circ}$ FR08/10, Agosto, Madrid, International Organization of Securities Commissions, 22 pp. [formato electrónico disponível em <https://www.iosco.org/library/pubdocs/pdf/IOSCOPD332.pdf $>$ ]. 
IOSCO (2015): "Mechanisms for Trading Venues to Effectively Manage Electronic Trading Risks and Plans for Business Continuity", Report of the Board of IOSCO, n. ${ }^{\circ}$ CR03/2015, Abril, Madrid, International Organization of Securities Commissions, 48 pp. [formato electrónico disponível em <https://www.iosco.org/library/pubdocs/pdf/IOSCOPD483.pdf $>$ ].

Johansson, Henrik (2013): High Frequency Trading: Market abuse and how to reestablish confidence in the market?, Jönköping, Master thesis within Economics, Jönköping International Business School, Jönköping University.

Johnson, Neil e ZHAO, Guannan (2012): "Brave new world: quantifying the new instabilities and risks arising in subsecond algorithmic trading", Foresight Driver Review, n. ${ }^{\circ}$ DR 27, 23 de Outubro, Londres, UK Government's Foresight Project, The Future of Computer Trading in Financial Markets, Government Office for Science, 45 pp. [formato electrónico disponível em <www.foresight.gov.uk>].

KaminsKa, Izabella (2009): "High frequency trading in Europe", Financial Times Alphaville Blog, 24 de Julho, http://www. ft.com/home/europe [formato electrónico disponível em <http://ftalphaville.ft.com/2009/07/24/63651/high-frequency-trading-in-europe $/>$.

- (2011): "Algo trading and the Nymex", Financial Times Alphaville Blog, 4 de Março, http://www.ft.com/home/europe [formato electrónico disponível em <http://ftalphaville. ft.com/2011/03/04/505021/algo-trading-and-the-ny$\operatorname{mex} />$ ].

Keller, Andrew J. (2012): "Robocops: Regulating High Frequency Trading After the Flash Crash of 2010", em Obio State Law Journal, Vol. 73, n. ${ }^{\circ}$ 6, pp. 1457-1483.

KIRILENKO, Andrei A. (2013): "Moore's Law versus Murphy's Law: Algorithmic Trading and Its Discontents", em Journal of Economic Perspectives, Vol. 27, n. ${ }^{\circ}$ 2, Primavera, pp. 51-72.

Kumiega, Andrew e Van Vliet, Benjamin Edward (2012): "Automated Finance: The Assumptions and Behavioral Aspects of Algorithmic Trading", em Journal of Behavioral Finance, Vol. 13, n. ${ }^{\circ}$ 1, pp. 51-55. 
Kunz, Karen e Martin, Jena (2015): "Into the Breech: The Increasing Gap between Algorithmic Trading and Securities Regulation", em Journal of Financial Services Research, Vol. 47, n. ${ }^{\circ} 1$, pp. 135-152.

LEWIs, Michael (2014): Flash Boys, trad. em português de Jorge Leonardo, Alfragide, Lua de Papel (1. ${ }^{a}$ ed., em inglês, 2014).

LiIKanen, Erkki [Presidente], Bänziger, Hugo, Campa, José Manuel, Gallois, Louis, Goyens, Monique, Krahnen, Jan Pieter, Mazzuccheldi, Marco, Sergeant, Carol, Tuma, Zdenek, Vanhevel, Jan e WijfFels, Herman (2012): High-level Expert Group on reforming the structure of the EU banking sector - final report, Bruxelas, 2 de Outubro de 2012 [formato electrónico disponível em <http://ec.europa.eu/ internal_market/bank/docs/high-level_expert_group/ liikanen-report/final_report_en.pdf>].

Menkveld, Albert J. (2011): "Electronic trading and market structure", Foresight Driver Review, n. ${ }^{\circ}$ DR 16, 10 de Agosto, Londres, UK Government's Foresight Project, The Future of Computer Trading in Financial Markets, Government Office for Science, 19 pp. [formato electrónico disponível em <www.foresight.gov.uk $>$ ].

Merli, Roberto, Massa, Ilaria e Lucchettr, Maria Claudia (2014): "High Frequency Trading: Technology, Regulation and Ethical Issues", em Achievements and challenges of commodity science in the age of globalization, org. por СНосно́,, Andrzej e Szakiel, Jerzy, Carcóvia, Polish Society of Commodity Science, pp. 179-192.

Mitra, Gautam, Di Bartolomeo, Dan, Banerjee, Ashok e Yu, Xiang (2011): "Automated Analysis of News to Compute Market Sentiment: Its Impact on Liquidity and Trading", Foresight Driver Review, n. ${ }^{\circ}$ DR 8, 20 de Julho, Londres, UK Government's Foresight Project, The Future of Computer Trading in Financial Markets, Government Office for Science, 29 pp. [formato electrónico disponível em $<$ www.foresight.gov.uk>].

NiciejewskA, Katharina (2015): Dark Pools and Flash Trading: New trends in Equity Trading?, Hamburgo, Anchor Academic Publishing. 
NoDA, Margareth (2010): Acesso eletrônico e tendências para a intermediação no mercado de valores mobiliários, São Paulo, Dissertação de Mestrado na Faculdade de Direito da Universidade de São Paulo, Biblioteca Digital de Teses e Dissertações da USP, 92 pp [formato electrónico disponível em <http://www.teses.usp.br/teses/disponiveis/2/2132/ tde-01122010-134441/en.php>].

O'Hara, Maureen (2015): "High frequency market microstructure", em Journal of Financial Economics, Vol. 116, n. ${ }^{\circ}$ 2, Maio, pp. 257-270.

Patterson, Scott (2013): Dark Pools: The Rise of the Machine Traders and the Rigging of the U.S. Stock Market, Nova Iorque, Crown Business.

Quelhas, José Manuel (2012a): "Dos objectivos da União Bancária", em Boletim de Ciências Económicas, Vol. LV, 2012, pp. 235-298.

- (2012b): "Sobre a criação do Comité Europeu de Risco Sistémico", em Estudos em Homenagem ao Prof. Doutor Aníbal Almeida, org. por Avelãs Nunes, António José, CunHA, Luís Pedro e Martins, Maria Inês de Oliveira, Coimbra, Boletim da Faculdade de Direito, Studia Iuridica, 107, Ad Honorem, 7, Coimbra Editora, pp. 877-899.

- (2012c): Sobre as crises financeiras, o risco sistémico e a incerteza sistemática, Coimbra, Almedina.

— (2013a): "Nótulas sobre a reforma do sector bancário da União Europeia após a crise financeira de 2007”, em Boletim de Ciências Económicas, Vol. LVI, 2013, pp. 473-549.

- (2013b): "Quem é que a austeridade cura? Quem é que a austeridade mata? - Releitura dos memoranda entre a troika e os PIGS", em $A$ austeridade cura? A austeridade mata?, org. por Ferreira, Eduardo Paz, Lisboa, Lisboa Law Scholl Editions, AAFDL, pp. 845-868.

- (2014): "Especificidades e vicissitudes do mecanismo de resolução bancária em Portugal: do Memorando de Entendimento de 2011 à Directiva 2014/59/UE e ao Regulamento (UE) n. ${ }^{\circ} 806 / 2014$ ", em Boletim de Ciências Económicas, Vol. LVII, Volume de Homenagem ao Professor Doutor António Avelãs Nunes, 48 pp. [no prelo]. 
Scholtus, Martin, van DijK, Dick e Frijns, Bart (2014): "Speed, algorithmic trading, and market quality around macroeconomic news announcements", em Journal of Banking and Finance, Vol. 38, pp. 89-105.

SornetTe, Didier e VON DER BeCKE, Susanne (2011): "Crashes and High Frequency Trading. An evaluation of risks posed by high-speed algorithmic trading ", Foresight Driver Review, n. ${ }^{\circ}$ DR 7, 10 de Agosto, Londres, UK Government's Foresight Project, The Future of Computer Trading in Financial Markets, Government Office for Science, 26 pp. [formato electrónico disponível em <www.foresight. gov.uk $>$ ].

Tenreiro, Mário Paulo e FrançA, Miguel (2012): “Artigo 290. do Tratado sobre o Funcionamento da União Europeia", em Tratado de Lisboa Anotado e Comentado, org. por PORTO, Manuel Lopes e AnAstácio, Gonçalo, Coimbra, Almedina, pp. 1039-1043.

The Government Office for ScIEnce (2012): "The Future of Computer Trading in Financial Markets", Final Project Report, 23 de Outubro, Londres, UK Government's Foresight Project, The Future of Computer Trading in Financial Markets, Government Office for Science, 178 pp. [formato electrónico disponível em <www.foresight.gov.uk>].

VAn Vliet, Benjamin Edward (2012): Systematic Finance: Essays on Ethics, Methodology and Quality Control in High Frequency Trading, Chicago, UMI Dissertations Publishing, Illinois Institute of Technology.

Zigrand, Jean-Pierre, Shin, Hyun Song e BeunZa, Daniel (2011): "Feedback effects and changes in the diversity of trading strategies", Foresight Driver Review, n. ${ }^{\circ}$ DR 2, 31 de Agosto, Londres, UK Government's Foresight Project, The Future of Computer Trading in Financial Markets, Government Office for Science, 24 pp. [formato electrónico disponível em <www.foresight.gov.uk>].

Zook, Matthew e Grote, Michael H. (2014): “The Microgeographies of Global Finance: High Frequency Trading and the Construction of Information Inequality", Fevereiro, 24 pp. [formato electrónico disponível em <SSRN: http: //ssrn.com/abstract $=2401030>$ ]. 
Resumo: O artigo examina o impacto da «negociação algorítmica de alta frequência» sobre o sistema financeiro, à luz do trade-off entre a eficiência e a estabilidade, dando particular ênfase ao problema designado por «demasiado rápido para falir».

Palavras-chave: negociação algorítmica de alta frequência; "demasiado rápido para falir".

\section{High-frequency trading (HF'T)}

Abstract: The article examines the impact of high frequency trading on the financial system in light of the trade-off between efficiency and stability, with particular emphasis on the issue usually known as "too fast to fail".

Keywords: high-frequency trading; "too fast to fail".

José Manuel Quelhas

Faculdade de Direito da Universidade de Coimbra 